\title{
Dimensões palpebrais antes e após blefaroplastia superior - Avaliação quantitativa
}

\author{
Eyelid measures beforeand after upperblepharoplasty-Quantitative evaluation
}

\author{
Silvana Artioli Schellini ${ }^{1}$ \\ Rony Carlos Preti ${ }^{2}$ \\ Ricardo Kazuhito Yamamoto ${ }^{2}$ \\ Carlos Roberto Padovani ${ }^{3}$ \\ Carlos Roberto Pereira Padovan ${ }^{4}$
}

\section{RESUMO}

Objetivo: Quantificar, usando o sistema de imagem digital, medidas palpebrais antes e após a cirurgia de blefaroplastia superior. Métodos: Foram avaliadas 18 pálpebras de 9 pacientes atendidas no HC da FMB - UNESP, comidade entre 40 a 75 anos, do sexo feminino, portadoras de dermatocálase. Foram obtidas fotografias das pacientes antes e após 60 dias da blefaroplastia da pálpebra superior. As imagens foram transferidas para um computador e analisadas pelo programa Scion Image Frame Grabber. Os parâmetros avaliados foram: a altura da fenda palpebral em posição primária do olhar, altura do sulco palpebral superior e o ângulo palpebral lateral antes e depois de 60 dias da realização da cirurgia de blefaroplastia superior. Resultados: Após a cirurgia, houve aumento da altura da fenda palpebral e do sulco palpebral superior. Contudo, o ângulo palpebral lateral não se alterou. Conclusão: A posição palpebral se altera após a blefaroplastia e o processamento de imagens digitais possibilita quantificar estas alterações, mensurando os resultados obtidos com a cirurgia.

Descritores: Blefaroplastia; Pálpebras/cirurgia; Medidas; Processamento de imagem assistida por computador; Feminino

\section{INTRODUÇÃO}

Dermatocálase refere-se a excesso de pele na pálpebra superior, inferior, ou ambas. Embora esteatoblefaro descreva mais especificamente a presença isolada de gordura orbital herniada, dermatocálase pode também incluir a presença de excesso de gordura e tecido muscular hipertrófico nas pálpebras $^{(1)}$. Socialmente, indivíduos com blefaroptose e dermatocálase podem ser vistos negativamente ${ }^{(2)}$, sendo a correção deste problema muito procurada atualmente.

O curso natural da afecção é o aumento gradual do peso de ambas as pálpebras superiores com o envelhecimento, que poderá provocar déficit de acuidade visual central ou diminuição do campo visual, causado por obstrução mecânica do campo de visão, alteração da cosmética das pálpebras, cefaléia frontal e excessiva fadiga à leitura, pelo uso crônico da musculatura frontal para vencer a obstrução visual ${ }^{(1)}$.

Blefaroplastia é o procedimento cirúrgico de escolha para corrigir ambos os aspectos, funcionais e cosméticos, relacionados a dermatocálase ${ }^{(1,3)}$.

A grande maioria dos estudos sobre a posição palpebral é qualitativa, ou feita utilizando-se réguas ou compassos. Porém, nos últimos anos, a literatura vem se enriquecendo de medidas quantitativas, principalmente pela possibilidade do processamento digital das imagens ${ }^{(4-5)}$, procedimento não invasivo e bastante preciso ${ }^{(4)}$. 
Com o aumento do interesse em pesquisar e analisar imagens, um programa para processamento destas permite que se afiram medidas de parâmetros palpebrais, tais como posição relativa do canto interno e externo, delineamento de curvas, posição das pálpebras ${ }^{(5-6)}$.

Porém, este tipo de pesquisa ainda foi pouco realizado, havendo apenas um estudo relacionado à blefaroplastia ${ }^{(6)}$, o que motivou a realização deste trabalho, que teve por objetivo avaliar quantitativamente medidas palpebrais em portadores de dermatocálase, antes e após a cirurgia de blefaroplastia superior.

\section{MÉTODOS}

Foram avaliados prospectivamente nove indivíduos (18 pálpebras), todos do sexo feminino, atendidos no HC da FMB UNESP, com idade entre 40 a 75 anos, portadores de dermatocálase, antes e depois de 60 dias de blefaroplastia da pálpebra superior. A técnica cirúrgica, assim como o cirurgião, foram mantidos constantes.

Os critérios de inclusão para participação do estudo foram: não apresentar outra patologia ou cirurgia óculo-palpebral prévia e a concordância do paciente em participar da pesquisa.

\section{Método de avaliação}

Todas as pacientes foram documentadas usando máquina fotográfica Asahi-Pentax K1000, com lente macro 50. O filme utilizado para revelação das imagens foi o Ektachrome ASA 100 da Kodak para diapositivos.

As fotografias foram feitas sempre pelo mesmo fotógrafo, conservando-se constantes à distância entre o paciente e a máquina, com a paciente olhando para o infinito, tomando-se as imagens imediatamente antes da cirurgia e 60 dias após a mesma.

As imagens obtidas foram escaneadas e transferidas para computador AMD-Athlon, com sistema operacional Windows XP 256 megabites de memória RAM. Utilizou-se o sistema Scion Image Frame Grabber para processamento das imagens digitalizadas.

Os parâmetros avaliados pelo "software" foram:

1. Altura da fenda palpebral (FP);

2. Altura do sulco palpebral superior (SPS);

3. Ângulo palpebral lateral (APL).

Os valores foram obtidos de cada olho, da seguinte forma:

1) A FP foi medida traçando-se uma linha reta da pálpebra superior até a inferior, passando-se pelo centro da pupila (Figura 1).

2) O SPS foi medido traçando-se uma linha reta do ponto central palpebral superior, com início no sulco propriamente dito, até a borda ciliar superior (Figura 2).

3) O ângulo palpebral lateral foi estimado traçando-se uma reta que unia o limbo temporal superior e inferior e outras duas com o vértice localizado no canto externo. A união destas linhas forma um triângulo, onde o ápice é o canto externo e a base faceia o limbo corneal (Figura 3).

4) Unidade de medida utilizada: na imagem do pré-operatório de cada olho, uma linha horizontal foi traçada de limbo a limbo, passando pelo centro da pupila. A medida obtida foi convencionada como sendo 1 (uma) unidade arbitrária e usada para a avaliação relativa das medidas deste estudo (Figura 4).

A partir de então, as medidas das variáveis (FP, SPS e APL) foram comparadas com a unidade arbitrária adotada e registradas.

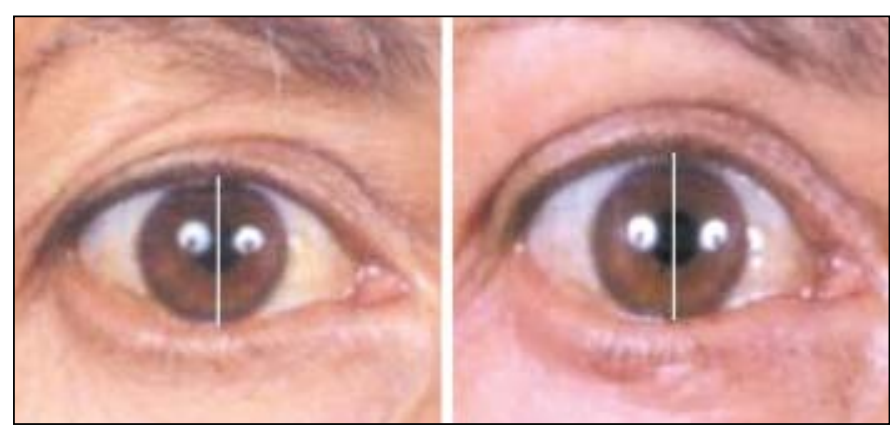

Figura 1 - Representação da medida da altura da fenda palpebral, obtida por uma linha que une a margem superior e inferior, passando pela pupila. À esquerda, paciente antes da cirurgia e à direita, após.

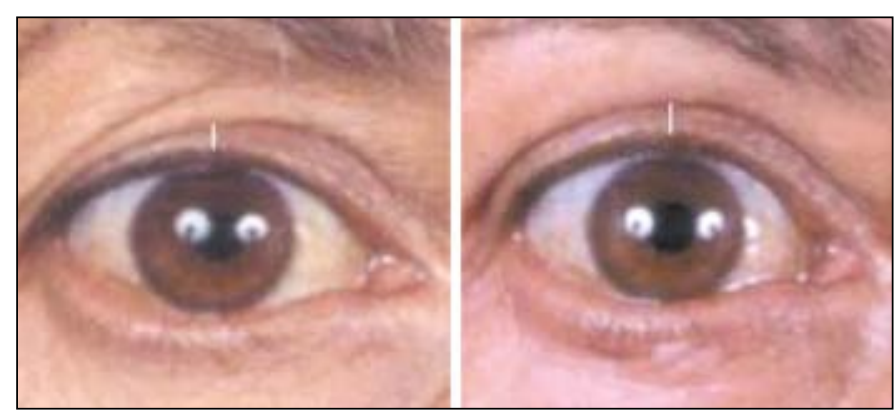

Figura 2 - Representação da medida do sulco palpebral superior, obtida por uma linha que une a margem superior e o sulco palpebral, na altura da pupila. À esquerda, paciente antes da cirurgia e à direita, após.

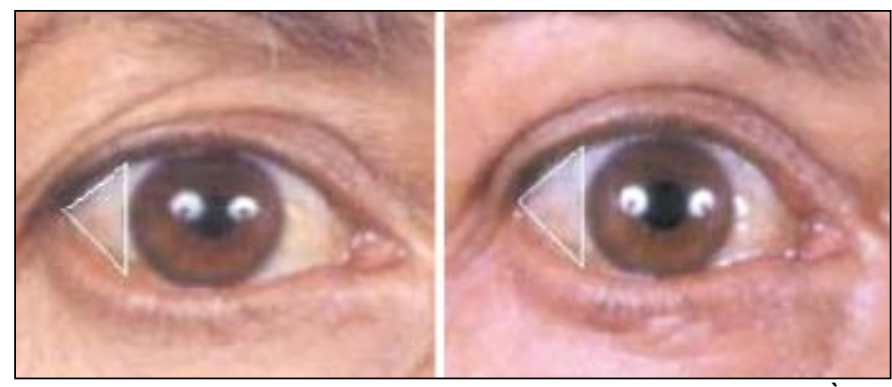

Figura 3 - Representação da medida do ângulo palpebral lateral. À esquerda, paciente antes da cirurgia e à direita, após.

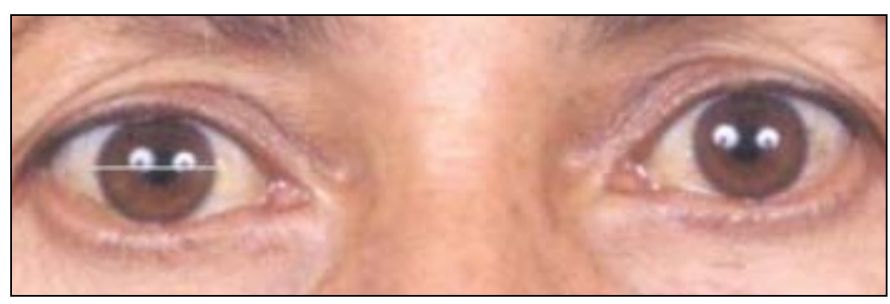

Figura 4 - Demonstração da medida relativa utilizada para a comparação das avaliações do estudo 
Os dados obtidos foram submetidos à avaliação estatística, usando-se o Teste t de Student para avaliação de amostras dependentes.

\section{Técnica cirúrgica}

Foi realizada a blefaroplastia superior convencional, com o paciente em decúbito dorsal horizontal e demarcação elíptica da quantidade de pele a ser excisada. O limite superior da elipse foi demarcado a $10 \mathrm{~mm}$ da margem inferior do supercílio e o limite inferior a $8 \mathrm{~mm}$ da linha dos cílios. Após a anestesia subcutânea na pálpebra superior com Xilocaína ${ }^{\circledR}$ a $2 \%$ com vasoconstrictor, a área demarcada foi removida, juntamente com o tecido subcutâneo e parte do músculo orbicular préseptal. Posteriormente, o septo orbitário foi aberto e a gordura orbitária redundante, ressecada e cauterizada. Por último, foi revista a hemostasia e a ferida cirúrgica foi suturada com Mononylon 6-0 (Ethicon), com pontos contínuos.

\section{RESULTADOS}

Na comparação das medidas da FP e do SPS no pré e no pós-operatório, observou-se aumento significativo destas duas variáveis após a cirurgia. Em relação ao ângulo palpebral lateral, não se evidenciou significância, quando comparadas as medidas do pré e do pós-operatório (Quadro 1).

\section{DISCUSSÃO}

O uso do processamento digital de imagens possibilita refinadas análises quantitativas de parâmetros oculoplásticos que podem ter importância clínica e cirúrgica ${ }^{(4)}$.

Digitalizar uma imagem significa atribuir valores espaciais (x, y) e de luminância aos pontos (pixeis) que compõem a figura. Uma vez digitalizada, a imagem pode ser processada por programas específicos que, ao manipularem matematicamente os pixeis, permitem o delineamento de bordas, filtragem de determinadas características e, evidentemente, a mensuração de qualquer parâmetro linear ou curvo da mesma ${ }^{(7-8)}$.

As imagens digitalizadas, ao serem processadas pelo programa Scion Image Frame Grabber, permitem mensurações de vários dados clínicos, como a medida angular, que seria imprecisa ou até impossível se utilizados instrumentos simples.

A síndrome do olho seco é o efeito adverso mais comum após a cirurgia de blefaroplastia ${ }^{(9-10)}$. No presente estudo, a FP aumentou de valor após a cirurgia, o que tende a gerar maior exposição corneana, contribuindo para o desenvolvimento da síndrome do olho seco, pois uma área de maior evaporação do filme lacrimal estaria ocorrendo. Contudo, ainda há controvérsias a este respeito, alguns defendem a opinião de que a blefaroplastia possa representar componente efetivo no tratamento de pacientes com dermatocálase e sintomas de olho seco ${ }^{(11)}$.

Segundo as observações deste estudo, houve aumento da FP e do SPS após a cirurgia. Porém, outro estudo que também avaliou quantitativamente imagens digitais mostrou que estes parâmetros não são afetados pela blefaroplastia ${ }^{(6)}$. A diferença entre os estudos pode ser decorrente de diferenças na técnica operatória.

Com a precisão de medidas de resultados que os softwares oferecem, os cirurgiões deparar-se-ão com dados mais confiáveis e poderão avaliar com segurança se determinada técnica cirúrgica tem trazido bons resultados. Também poderão melhor estimar as mudanças necessárias para melhoria de sua aplicabilidade, amenizando ou até evitando as complicações.

Outro aspecto seria o suprimento das expectativas póscirúrgicas dos pacientes, de forma satisfatória e com maior probabilidade de acerto.

Assim, as avaliações realizadas permitiram concluir que o processamento de imagens digitais possibilita avaliações quantitativas de parâmetros palpebrais nas cirurgias de dermatocálase, o que poderá ser útil para aplicação em estudos futuros.

\section{ABSTRACT}

Purpose: To evaluate eyelid measures before and after upper blepharoplasty surgery using a digital image system. Methods: Eighteen eyelids of 9 patients with dermatochalasis were evaluated at the Hospital das Clínicas de Botucatu - UNESP regarding eyelid measures before and after upper blepharoplasty. All patients were female, aged between 40 and 75 years. The images were acquired by a photographic camera, transferred to a computer and then analyzed by the Scion Image Frame Grabber program. The evaluated parameters were upper eyelid position in primary gaze position, upper palpebral sulcus and lateral eyelid angle. The measures were obtained before and after 60 days of upper dermatochalasis surgery. Results: After the surgery, there was an increase of upper eyelid position and upper palpebral sulcus; the lateral palpebral angle did not show any alteration. Conclusion: Eyelid position changes after blepharoplasty and digital image processing provides quantitative measures of the eyelid, allowing a better quantification of the surgical results.

Keywords: Blepharoplasty; Eyelids/surgery; Measures; Image processing, computer-assisted; Female

\begin{tabular}{|lrrr|}
\hline Quadro 1 - Média e desvio padrão das variáveis estudadas e resultado do teste estatístico da comparação dos momentos de avaliação \\
\cline { 2 - 3 } Variável & \multicolumn{2}{c}{ Momento } & Resultado do teste estatístico \\
Fenda palpebral & $0,7494 \pm 0,1467$ & $0,7989 \pm 0,1375$ & $2,37(\mathrm{P}<0,05)$ \\
Sulco palpebral superior & $0,1239 \pm 0,0757$ & $0,2544 \pm 0,0719$ & $3,05(P<0,05)$ \\
Ângulo palpebral lateral & $65,5790 \pm 12,0340$ & $69,9060 \pm 9,5275$ & $1,70(P>0,05)$ \\
\hline
\end{tabular}




\section{REFERÊNCIAS}

1. DeAngelis DD, Carter SR, Seiff SR. Dermatochalasis. Int Ophthalmol Clin. 2002;42(2):89-101. Review.

2. Warwar RE, Bullock JD, Markert RJ, Marciniszyn SL, Bienenfeld DG. Social implications of blepharoptosis and dermatochalasis. Ophthal Plast Reconstr Surg. 2001;17(4):234-40.

3. Fagien S. Advanced rejuvenative upper blepharoplasty: enhancing aesthetics of the upper periorbita. Plast Reconstr Surg. 2002;110(1):278-91; discussion 292.

4. Cruz AA, Coelho RP, Baccega A, Lucchezi MC, Souza AD, Ruiz EE. Digital image processing measurement of the upper eyelid contour in Graves disease and congenital blepharoptosis. Ophthalmology. 1998;105(5):913-8.

5. Sampaio Jr AA, Hoyama E, Schellini SA, Cruz AAV, Padovani CR. Avaliação do tempo de fechamento e abertura palpebral durante o piscar reflexo em indivíduos normais. Arq Bras Oftalmol. 2001;64(4):64-114.
6. Starck WJ, Griffin JE Jr, Epker BN. Objective evaluation of the eyelids and eyebrows after blepharoplasty. J Oral Maxillofac Surg. 1996;54(3):297-302; discussion 302-3.

7. Souza AD, Ruiz EE, Cruz AA. Palpebral fissure morphology segmentation and measurement using image processing. IEEE Eng Med Biol Mag. 2000; 19(1):114-9.

8. Cruz AAV, Baccega A. Análise bidimensional computadorizada da fenda palpebral. Arq Bras Oftalmol. 2001;64(1):13-9.

9. Burke AJ, Wang T. Should formal ophthalmologic evaluation be a preoperative requirement prior to blepharoplasty? Arch Otolaryngol Head Neck Surg. 2001;127(6):719-22.

10. Lessa SF, Elena EO, Araújo MRC, Pitanguy I. Modificações anatômicas da fenda palpebral após blefaroplastia. Rev Bras Cir.1997;87(4):179-88.

11. Vold SD, Carroll RP, Nelson JD. Dermatochalasis and dry eye. Am J Ophthalmol. $1993 ; 115(2): 216-20$.

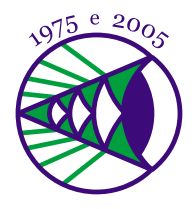

\section{Congresso Brasileiro de Oftalmologia}

3 a 6 de setembro de 2005 - Centro de Convenções do Ceará - Fortaleza - Ceará

\section{COMISSÃO EXECUTIVA}

Presidente: Leiria de Andrade Neto

Vice-Presidentes: Emilson Barros de Oliveira Luciano Alencar Newton Leitão de Andrade Waldo Pessoa
Secretários: Valter Justa Islane Verçosa Germano Andrade

Tesoureiros: Renato Stênio T. Rodrigues Antonio Augusto Matos Pires Márcia Medeiros

\section{VALORES DE INSCRIÇÃO}

\begin{tabular}{|c|c|c|c|c|}
\hline Categoria & $\begin{array}{c}\text { Até } \\
\text { 31/03/05 } \\
\text { (R\$) }\end{array}$ & $\begin{array}{c}\text { De 01/04/05 } \\
\text { a 31/05/05 } \\
\text { (R\$) }\end{array}$ & $\begin{array}{c}\text { De 01/06/05 } \\
\text { a 31/07/05 } \\
\text { (R\$) }\end{array}$ & $\begin{array}{l}\text { No } \\
\text { Local } \\
\text { (R\$) }\end{array}$ \\
\hline Sócios CBO & 300,00 & 345,00 & 415,00 & 550,00 \\
\hline Não Sócios & 620,00 & 700,00 & 800,00 & $1.000,00$ \\
\hline Residente e Acadêmicos & 150,00 & 175,00 & 210,00 & 280,00 \\
\hline Acompanhantes & 80,00 & 90,00 & 100,00 & 120,00 \\
\hline Passanorte Sócios CBO & 100,00 & 100,00 & 100,00 & 150,00 \\
\hline Passaporte Não Sócios & 125,00 & 125,00 & 125,00 & 200,00 \\
\hline $\begin{array}{l}\text { Números para concorrer ao } \\
\text { sorteio de um carro* }\end{array}$ & 3 chances & 2 chances & 1 chance & 1 chance \\
\hline
\end{tabular}

*Inscreva-se no evento e concorra a um carro $0 \mathrm{Km}$

Atenção

Data limite para envio de Temas Livres e Pôsteres: 15/03/2005

Secretaria Executiva

Arx Produções \& Eventos

Tel.: (85) 4011-1572 - Fax: (85) 4011-1573

E-mail: cbo2005@arxweb.com.br
Agência de Turismo Oficial

Tour du Monde

Tel.: (85) 3462-5863 - Fax: (85) 3462-5899 E-mail: contato@tourdumonde.tur.br

\section{Visite nosso site: www.cbo2005.com.br}

Faça sua inscrição pela internet e concorra a uma passagem aérea para o evento 